OPEN ACCESS

Edited by:

Juan Gilbert

University of Florida, United States

Reviewed by:

Karina Liles,

Claflin University, United States

Curtis Cain,

Howard University, United States

Chris Crawford,

University of Alabama, United States

Edward Dillon,

Morgan State University,

United States

*Correspondence:

Michael Preuss

exquiri.michael@gmail.com

Samuel Merriweather

s_merriweather@tamu.edu

${ }^{\dagger}$ These authors share first authorship

Specialty section: This article was submitted to

STEM Education,

a section of the journal

Frontiers in Education

Received: 01 March 2021

Accepted: 09 June 2021

Published: 20 July 2021

Citation:

Preuss M, Merriweather S, Avila J, Butler-Purry K, Watson K, Walton S, Obiomon P, Pezold F, Murry J, Roth M,

Kelley J, Lamm H, Alves $M$ and Garcia S (2021) The Impacts of Global

Research and International

Educational Experiences on Texas

A\&M University System

LSAMP Participants.

Front. Educ. 6:674772

doi: 10.3389/feduc.2021.674772

\section{The Impacts of Global Research and International Educational Experiences on Texas A\&M University System LSAMP Participants}

\author{
Michael Preuss ${ }^{1 * t}$, Samuel Merriweather ${ }^{2 * t}$, John Avila ${ }^{2 t}$, Karen Butler-Purry ${ }^{3,4}$, \\ Karan Watson ${ }^{4}$, Shannon Walton ${ }^{2,3}$, Pamela Obiomon ${ }^{5,6}$, Frank Pezold ${ }^{7,8}$, Jasmine Murry ${ }^{5}$, \\ Michele Roth ${ }^{7}$, Judy Kelley ${ }^{9}$, Harriet Lamm ${ }^{2}$, Maria Alves ${ }^{10}$ and Sonia Garcia ${ }^{11}$ \\ for the Texas A\&M University System Louis Stokes Alliance for Minority Participation
}

${ }^{1}$ Exquiri Consulting, LLC, Amarillo, TX, United States, ${ }^{2}$ Texas A\&M University System LSAMP, Texas A\&M Engineering Experiment Station, College Station, TX, United States, ${ }^{3}$ Graduate and Professional School, Texas A\&M University, College Station, TX, United States, ${ }^{4}$ Department of Electrical and Computer Engineering, College of Engineering, Texas A\&M University, College Station, TX, United States, ${ }^{5}$ College of Engineering, Prairie View A\&M University, Prairie View, TX, United States,

${ }^{6}$ Department of Electrical and Computer Engineering, College of Engineering, Prairie View A\&M University, Prairie View, TX, United States, ${ }^{7}$ College of Science and Engineering, Texas A\&M University-Corpus Christi, Corpus Christi, TX, United States, ${ }^{8}$ Department of Life Sciences, College of Science and Engineering, Texas A\&M University-Corpus Christi, Corpus Christi, TX, United States, ${ }^{9}$ Killgore Research Center, West Texas A\&M University, Canyon, TX, United States, ${ }^{10}$ Halliburton Engineering Global Programs, College of Engineering, Texas A\&M University, College Station, TX, United States, ${ }^{11}$ Access and Inclusion Program, College of Engineering, Texas A\&M University, College Station, TX, United States

The Texas A\&M University System was one of the first six Louis Stokes Alliance for Minority Participation (LSAMP) awardees. All current members of the Alliance are part of the Texas A\&M University System. Many high impact practices (HIP) have been emphasized in the Alliance's 30 years of programming with Diversity/Global Learning as a focus in the last 14 years. Diversity/Global Learning has been supported in two formats on the Alliance campuses, through traditional study abroad programming and a College of Engineering initiative. Data presented were derived from a number of sources, project evaluation information regarding student perspectives and outcomes, survey research conducted by an independent party, and institutional data and online platforms accessed to assess student outcomes. Triangulation was completed between data sets. Results indicate both forms of programming were efficacious for underrepresented and first-generation students. Outcomes reported were substantial increases in awareness of and interest in graduate school, increases in cultural learning, confidence in travel outside the United States, learning relevant to major, commitment to continuing involvement with research, interest in another similar experience, and willingness to consider employment outside the U.S. Participants reported statistically significant growth in personal, professional, and research skills. They persisted, participated in additional study abroad experiences, and graduated at higher rates than their institutional peers with approximately $90 \%$ of informants indicating intention to consider graduate school in the future, over $40 \%$ indicating intent to attend immediately following undergraduate study, and $39.4 \%$ of $2007-2014$ participants enrolling in graduate school by the spring of 2021 . 
Programming described is replicable at and likely to be efficacious for a wide variety of institutions of higher education.

Keywords: LSAMP, high impact practice, study abroad, global learning, underrepresented minority students, firstgeneration college students, STEM

\section{INTRODUCTION}

“The Texas A\&M University System (TAMUS) Louis Stokes Alliance for Minority Participation (LSAMP) program... [focuses on] encouraging and supporting...underrepresented minority (URM) science, technology, engineering, and mathematics (STEM) majors at ... Alliance member" (Merriweather et al., 2017, p. 1) institutions. "Formally called TX LSAMP, the Alliance was one of the first six LSAMPs funded by NSF" (Merriweather et al., 2017, p. 1) in 1991. Since that time, TAMUS LSAMP has supported over 11,500 students "for one or more semesters of their undergraduate studies" (Merriweather et al., 2017, p. 1) and Alliance institutions have awarded over 22,000 STEM degrees to URMs. "Using a carefully conceived suite of opportunities specially designed for URM undergraduate students...the Alliance has" (Merriweather et al., 2017, p. 1) sought improvement of academic success for underrepresented students. Within the overall emphasis on academic success and advancement, programming offered and the number of member institutions have varied in the last 30 years.

Current TAMUS LSAMP member institutions are Texas A\&M University at College Station (TAMU), a Very High Research Activity institution in the Carnegie Classification System (Indiana University Center for Postsecondary Research, n.d.), Prairie View A\&M University (PVAMU), an Historically Black College and University (HBCU) (U.S. Department of Education, 2020), Texas A\&M University-Corpus Christi (TAMUCC), an Hispanic-Serving Institution (HSI) (National Center for Education Statistics, 2018), and Texas A\&M International University (TAMIU), an HSI (National Center for Education Statistics, 2018). TAMUCC's Carnegie classification is Doctoral Universities: High Research Activity. Both PVAMU and TAMIU are in the Master's Colleges and Universities Larger Program category (Indiana University Center for Postsecondary Research, n.d.). The material that follows will include data regarding students from TAMU, PVAMU, and TAMUCC as TAMIU is a recent addition to the Alliance and international programming was prohibited by COVID-19 in 2020.

The material presented addresses a high impact practice (HIP) in higher education (American Association of Colleges and Universities [AACU], n.d.; Kuh and O' Donnell, 2013) that has been emphasized within TAMUS LSAMP. This HIP is Diversity/Global Learning, "courses and programs that help students explore cultures, life experiences, and worldviews different from their own" (AACU, n.d.). Support of international programming became a project objective in 2007. Consciousness of globalization (Smith and Mitry, 2008), the increasingly international nature of engineering practice (Borri et al., 2007; Chan and Fishbein, 2009), and the personal, academic, intercultural, and career benefits reported for undergraduates participating in study abroad programming (Dwyer and Peters, 2005) motivated this action. Specifically, "employability skills such as interpersonal and communication skills, teamwork skills...problem solving and analytical skills" (Potts, 2015, p. 441) were in view as they had been reported "as the greatest perceived benefits" (Potts, 2015, p. 441) of participation in study abroad. "Career-related benefits such as future career prospects and increased motivation and passion for their chosen career direction" (Potts, 2015, p. 441) and intercultural learning (Kamdar and Lewis, 2015; Paras et al., 2019), which had also been identified with study abroad, were other motivating factors. The result was a series of programming emphases that have spanned 14 years.

Two different forms of study abroad programming were found to be efficacious for underrepresented and first-generation engineering students. Outcomes reported were substantial increases in awareness of and interest in graduate school, increases in cultural learning, confidence in travel outside the United States, learning relevant to major, commitment to continuing involvement with research, interest in another similar experience, and willingness to consider employment outside the United States. Participants also reported statistically significant growth in personal, professional, and research skills. They persisted, participated in additional study abroad experiences, graduated, and enrolled in graduate school at higher rates than their institutional and national peers.

\section{PEDAGOGICAL FRAMEWORK}

Three reports have been published that include information about international programming in TAMUS LSAMP. They are, in chronological order: 1) Garcia et al. (2017), 2) Merriweather et al. (2017), and 3) Preuss et al. (2020). The pedagogical framework for TAMUS LSAMP international programming prior to 2015 was not addressed in these publications but that after 2015 has been. A brief description of both frameworks will follow with the description of activity after 2015 referencing the earlier publications.

TAMUS LSAMP's first framework for Diversity/Global Learning was support of individual students in study abroad opportunities they sought out. The second, which continues to the present, was facilitation of a TAMU College of Engineering (COE) initiative offered annually and designed for underrepresented and first-generation students.

Support of study abroad opportunities officially became a project goal in November of 2007. By the summer of 2008 there were five TAMU and two TAMUCC students participating in international experiences in Singapore, Spain, 
and Mexico. Participation increased at a regular and steady pace from that point forward, with 10 students supported in 2011 in Spain, Mexico, El Salvador, and six locations in Brazil.

To promote Diversity/Global Learning TAMUS LSAMP instituted seminars for participants that described study abroad opportunities. These seminars were offered in two ways. Seminars specific to each Alliance member were held each year and the annual research symposium sponsored for TAMUS LSAMP participants included a presentation about study abroad opportunities. The seminars were intended to increase awareness of study abroad opportunities and included information to help students overcome institutional obstacles and hesitancy on their part or that of their parents, concerns especially relevant to underrepresented and first-generation college students (Brux and Frye, 2010). Evaluation data gathered from students at each institution over a five-year period confirmed the seminars were offered each year and showed that between $67 \%$ and $90 \%$ of the participants found these sessions at least somewhat helpful.

While seminars about study abroad opportunities continued within the Alliance, emphasis shifted in the 2014-2015 school year to support of the Engineering Learning Community Introduction to Research (ELCIR) program. ELCIR is a onecredit hour, introduction-to-research course that includes "a two-week, study-abroad research program implemented in a learning community pattern. Ten days of international instruction are completed at" (Preuss et al., 2020, p. 1) institutions of higher education in Merida, Yucatan that partner with TAMU. "ELCIR has three purposes: 1) to expose students to research early in their academic careers, 2) to introduce students to cultural differences and global challenges, and 3) to provide students with the basic tools to prepare them for future research opportunities within TAMU's College of Engineering research internship programs, especially study abroad internships" (Preuss et al., 2020, p. 2). Participation during the first two years was "limited to first generation college students and/or students from underrepresented populations who are associated with the Access and Inclusion program in the College of Engineering" (Preuss et al., 2020, p. 2) but was expanded to larger cohorts open to any freshman Engineering student following that.

One distinction between TAMUS LSAMP's initial and later emphasis on Diversity/Global Learning was the age of the participants. Prior to 2015, students funded for study abroad were exclusively juniors and seniors $(n=33)$. The 2014-2015 transition to supporting student participation in ELCIR included shifting to early career students, freshmen and sophomores. A second distinction between the two periods was the emphasis placed on research as a component of the student's international experience. From 2007 to 2014, students completing international opportunities focused primarily on completing courses at a university outside the United States. Of the 28 students funded for international experiences who provided feedback from 2007 to 2012, all but one reported taking classes but only four reported participating in a research endeavor as part of their time abroad. The one exception to class taking was a student who participated in a summer medical internship and the first report of research involvement as part of a TAMUS LSAMP international experience was four years into the initiative in 2012. ELCIR, though, was conceived as an opportunity that included exposing "students to research early in their academic careers" (Preuss et al., 2020, p. 2).

\section{LEARNING ENVIRONMENT}

Prior to 2015, students were encouraged to participate in study abroad opportunities recognized by their university. These, as has already been noted, were predominantly study at a university outside the United States. Only four of 28 informants in a fiveyear period participated in a research project as part of their study abroad programming.

In the 2014-2015 school year, TAMUS LSAMP shifted to supporting student participation in the ELCIR program. It "engages students at the beginning of their engineering education in four sets of experiences: 1) a hands-on research class, allowing students to identify their own research problem with the support of faculty and researchers, 2) international travel and two-week residence outside the United States, 3) engagement with highly experienced researchers and well-known research centers, and 4) a [five-page report regarding and] poster presentation of their research proposal results to peers, faculty, and administrators" (Preuss et al., 2020, p. 2). As noted in Preuss et al. (2020), the result is a combination of up to seven HIPs in one program.

"The intention of ELCIR is 'for underrepresented first generation ethnic minority students to be engaged in a research course" (Garcia et al., 2017, p.2). "Participant selection is based on the student's status as an underrepresented minority and/or as a first-generation college student, his/her grade point average and resume, and a response to a question about what $s /$ he expects to gain from participating in the project. A letter of recommendation from a faculty member [wa]s also requested and considered as part of the participant application" (Preuss, et al., 2020, p. 2) for the first 2 years. Since participants are early in their college careers, faculty could comment on little beyond class performance limiting the breadth of the recommendations and the practice of gathering faculty recommendations was discontinued beginning in ELCIR's third year.

A “one-credit course, ENGR 291 -Engineering Learning Community Introduction to Research, was added in 2016.... Inclusion of course credit has been maintained since that time. The initial course consisted of workshops regarding research, global competency, and travel preparation that were conducted with the ELCIR cohort in the spring of their freshman year. It has since been expanded to include more specificity in some areas and to accommodate several additional topics. These include introduction of the ELCIR Program purpose and goals, introduction to research topics, introduction to LSAMP/NSF sponsored responsibilities, research and research abroad expectations, [a] seminar on cultural competency, expectations [regarding] living with host families, [and] traveling/departure official documents' (Garcia et al., 2017, p. 3)" (Preuss et al., 2020, 
p. 2). The first year ELCIR participants stayed in a hotel but from 2016 on they have been placed with host families to increase cultural learning.

"The two-week international experience is a trip to Merida, Mexico where participants attend an introduction to research seminar (two hours per day), make visits to research sites and participate in research expeditions, receive hands-on experience in research labs, conduct their own research, visit cultural sites, and participate in cultural learning activities. The research course in the summer experience has been taught by 'Dr. Medina-Cetina and the vice president for research of Universidad Marista' (Garcia et al., 2017, p. 4)" (Preuss et al., 2020, p. 2). Growth of the program and expansion of partnerships in Yucatan resulted in the addition of Dr. John Walewski as a facilitator and a second host institution, the Politechnic University of Yucatan in 2017. Also, in 2017, Mexican students were incorporated in the program enabling multi-national student teams in activities and assignments. "Participants can select from a group of topic areas in which to conduct research. These are 'energy, coastal dynamics, logistics, aquifers and early warning system [s]' (Garcia et al., 2017, p. 2) which were chosen because faculty from TAMU collaborate with researchers in Yucatan in these areas" (Preuss et al., 2020, p. 2).

When they return to the U.S., TAMU "participants complete research reports and create research posters based on their investigations in Merida, Mexico. An online community is maintained as part of the project and used as a resource for exchanging materials, offering guidance, and then providing critiques when students are developing their research papers and presentations. Research posters are presented at TAMU COE in September each year" (Preuss et al., 2020, pp. 2-3).

\section{RESULTS TO DATE}

The material presented covers 13 years of programming for engineering students and is based on survey data. Several publications, as noted above, have been completed. The distinctive and notable contribution made here is consideration of 13 years of data rather than two- or four-year segments, inclusion of material from all applicable data sets, data regarding two distinct forms of international experience, consideration of outcomes for upper level and early career students, and presentation of long-term impact on participation in additional study abroad programming, persistence, graduation, and continuation to graduate school. The data included were drawn from project evaluation, with consideration of two different pedagogical frameworks, a research endeavor, and programmatic, institutional, and online student outcomes data. The primary emphasis was on obtaining information about and understanding the cumulative impact of study abroad experiences and ELCIR rather than the impact of various components of these opportunities. Surveys completed prior to 2015 had a participation rate just over $90 \%$. The ELCIR-specific survey responses have a $95 \%$ confidence level with a confidence interval of 4.29 as they were submitted by 91 of 110 participants in five distinct cohorts $(82.7 \%$ response rate).
The information presented is arranged chronologically. Material from before 2015 is presented first. It is based on self-reported data from juniors and seniors after they participated in traditional study abroad programming. That is followed by information provided by ELCIR participants who were almost entirely freshman and sophomores. Data gathering for project evaluation of ELCIR did not include pre-participation processes. However, Garcia et al.'s (2017) investigation of the first two years of programming did. Quantitative data from project evaluation underwent descriptive and tabular analysis while Garcia et al.'s (2017) data could support inferential statistics. Thus, this consideration addresses two forms of Diversity/Global Learning programming for students with similar backgrounds, all from the same institutions. These two populations provided responses regarding many of the same topics. That information is supplemented by insights from Garcia et al. (2017) which discusses a separate data set.

\section{Study Abroad Programming With Juniors and Seniors (2007-2014)}

Three surveys that had the entire TAMUS LSAMP participant population as the audience included questions about the impact of the seminars regarding study abroad. Some of the questions were deployed for two years and others for as many as 4 years.

Responses from two retrospective pre- and post-participation questions appear in Table 1 (informants were asked to recall and report their pre-participation stance). These questions addressed awareness of international education experiences and interest in them. They were multiple choice questions that employed customized Likert scales. The choices offered to the informants on the first were: 1) never heard anything about (NHAA), 2) only heard a little about (OHALA), 3) had some basic knowledge (HSBK), 4) had some understanding (HSU), and 5) had a good understanding (HGU). These were meant to assess levels of awareness. The choices for the second question, meant to assess interest, were: 1) never heard anything about, 2) not at all interested in (NAAI), 3) a little interested in (ALI), 4) interested in (II), and 5) very interested in (VII).

The customized Likert scales make statistical analysis impossible as the responses are not all related to the same construct and were nominal, for example never heard anything about and not at all interested do not address the same idea or represent a pattern with defined distance between the options. However, the results do indicate changes in awareness and interest resulting from exposure to one or more seminars about study abroad opportunities. More than $77 \%$ of respondents felt they had at least some understanding following participation when $24 \%$ of them reported this level of understanding as their prior state. Responses for interest were similar with selection of not at all interested dropping by $15.3 \%$, a little interested in dropping by $7.2 \%$, while interested in and very interested in increased by $8.1 \%$ and $23.0 \%$, respectively.

Questions specific to the seminars offered at the annual research symposium were also asked. A query that occurred only in 2010 asked how helpful the informants felt information about study abroad and international experiences 
TABLE 1 | Pre- and post-participation awareness of and interest in international educational experiences.

\begin{tabular}{|c|c|c|c|c|c|c|c|c|}
\hline Topic & $n$ & Period & NHAA & OHALA & HSBK & HSU & HGU & NR \\
\hline \multirow[t]{3}{*}{ Awareness of international educational/research experiences. } & 120 & Before & $10.8 \%$ & $35.0 \%$ & $29.2 \%$ & $14.2 \%$ & $10.0 \%$ & 0.8 \\
\hline & & After & $0.0 \%$ & $5.0 \%$ & $15.8 \%$ & $34.2 \%$ & $43.3 \%$ & $1.7 \%$ \\
\hline & $n$ & Period & NHAA & NAAI & $A L I$ & II & VII & NR \\
\hline \multirow[t]{2}{*}{ Interest in international educational/ research experiences. } & 222 & Before & $10.4 \%$ & $18.9 \%$ & $31.5 \%$ & $21.6 \%$ & $17.1 \%$ & 0.5 \\
\hline & & After & $0.9 \%$ & $3.6 \%$ & $24.3 \%$ & $29.7 \%$ & $40.1 \%$ & $1.4 \%$ \\
\hline
\end{tabular}

Note: $N R=$ no response. The awareness data are from three years. The interest data are from four years. It is possible that some individuals submitted more than one response for each if they were involved with TAMUS LSAMP programming for more than one year.

TABLE 2 | Impact of seminars about international education experiences.

\begin{tabular}{|c|c|c|c|c|c|c|c|}
\hline Topic & $n$ & NAA & $A L$ & SWT & A lot & AGD & NR \\
\hline Degree to which on campus LSAMP meetings/workshops/seminars contributed to interest. & 173 & $11.0 \%$ & $12.7 \%$ & $32.4 \%$ & $26.6 \%$ & $16.8 \%$ & $0.6 \%$ \\
\hline Degree to which LSAMP symposium workshops contributed to interest. & 104 & $16.3 \%$ & $17.3 \%$ & $26.9 \%$ & $14.4 \%$ & $20.2 \%$ & $4.8 \%$ \\
\hline
\end{tabular}

Note: $N A A=$ not at all, $A L=$ a little, $S W T=$ somewhat, $A G D=$ a great deal, $N R=$ no response .

TABLE 3 | Change in perspective reported by study abroad participants.

\begin{tabular}{|c|c|c|c|c|c|c|}
\hline Topic & $n$ & SD & D & NAD & A & SA \\
\hline Learned about the culture of the area that I visited. & 28 & - & - & - & $14.3 \%$ & $85.7 \%$ \\
\hline The experience increased confidence in ability to travel abroad. & 28 & - & - & $3.6 \%$ & $3.6 \%$ & $92.9 \%$ \\
\hline Would consider a job in another country as a result of the study abroad experience. & 28 & $3.6 \%$ & - & $7.1 \%$ & $21.4 \%$ & $67.9 \%$ \\
\hline Knowledge and understanding of concepts in major enhanced during the international experience. & 28 & - & - & $7.1 \%$ & $28.6 \%$ & $64.3 \%$ \\
\hline Interested in participating in a similar international experience. & 28 & - & - & $3.6 \%$ & $10.7 \%$ & $85.7 \%$ \\
\hline Able to participate because of the support provided by LSAMP. & 28 & - & - & - & - & $100 \%$ \\
\hline
\end{tabular}

Note: $S D=$ strongly disagree, $D=$ disagree, $N A D=$ neither agree or disagree, $A=$ agree, and $S A=$ strongly agree.

was to them. Forty-six of the 60 participants responded, a $76.7 \%$ response rate $(95 \%$ confidence with a 7.04 interval). Of them, $53.3 \%$ felt the seminar was very helpful and $23.4 \%$ that it was helpful. An additional 15.0\%, 9 persons, found is slightly helpful. While only asked 1 year, almost $92 \%$ of the 46 respondents that year reported that the symposium seminar was slightly to very helpful. Responses from two prompts, one focused on the LSAMP programming and the other specific to the symposium seminars appear in Table 2. These multiple-choice questions used the same customized Likert scale (see explanation in the table).

Like above, the use of a nominal Likert scale prevented statistical analysis. Yet, the responses from students across four years suggest that the on-campus seminars, which would have been prepared by local personnel and included opportunities specific to the institution, had a greater impact than the general interest sessions presented at the symposium although $61.5 \%$ of the informants found the symposium workshops about international experiences contributed at least somewhat to their interest.

A series of six questions was asked of participants from 2010 to 2013 (Table 3). This information represents the earliest TAMUS LSAMP efforts to gather specifics about impacts of international experiences. The question set remained uniform for the entire period and the students participating were recruited as juniors and seniors. Four of these questions were retained for 2015 on but, as noted above, the audience and programming experienced were different at that time.

Only one student disagreed at any level with a prompt. That was for considering employment outside the United States. The ratings submitted facilitate a rank ordering by percent agreement. Items with the higher percentage of strongly agree responses were listed first in the case of ties.

1. Able to participate because of LSAMP support (100\%). Learned about the culture of area visited (100\%).

2. Increased confidence in ability to travel abroad (96.4\%). Would like another similar experience (96.4\%).

3. Enhanced understanding of major (92.9\%).

4. Would consider employment outside the United States (89.3\%).

These responses from 28 students across a four-year period support the efficacy of study abroad and, in this case, its effect on LSAMP participants. Many of the students would have been from underrepresented groups but determination of the exact percentage is not possible as ethnic and racial identity information was not solicited on the surveys during this period. 


\section{ELCIR Programming with Freshmen and Sophomores (2015-2019)}

From 2015 through 2019, TAMUS LSAMP sponsored involvement in ELCIR. This was also planned for summer of 2020 but prevented by the COVID-19 pandemic. Students participating in the programming were asked a consistent set of questions on a post-participation survey. These included demographic information and 14 other queries. Ninety-one students from a total cohort of 110 submitted responses. The following is a compendium of results from all known sources including previously unpublished material from evaluation data.

\section{Informant Demographics and Experiential Background}

ELCIR survey informants provided demographic data. There were 44 females, 45 males, and two persons who did not categorize themselves. Of these, 82 identified as Hispanic and eight as non-Hispanic (one no response). These individuals thought of themselves as African American $(n=7)$, Native American $(n=6)$, Other $(n=8)$, and White $(n=69)$ (four no response; Hispanic/Latino was not included as a racial category). When completing the survey in the fall following their summer ELCIR experience, they reported academic classification as freshmen $(n=1)$, sophomores $(n=80)$, juniors $(n=8)$, and seniors $(n=1)$ (also one no response). Preuss et al. (2020) presents a comparison of these figures to the overall cohort of LSAMP-funded participants in ELCIR programming during the same period. The outcome of that comparison was recognition of informants exhibiting "a slight shift toward females when compared to the overall cohort. The ethnic identity of the survey respondents was similar to that of the overall cohort, the majority of the respondents (89.0\%) identified as Hispanic, although this shows there was a slight oversampling of non-Hispanics. The distribution across races was similar.... Overall, the sample parallels the cohort with limited variation which was most pronounced in proportion of females to males, $5 \%$ more females in the sample, and in respect to underrepresentation of persons identifying as Native Americans/Alaska Natives" (p. 7).

Participants were asked if they had experience with international travel and study abroad as this had the potential to impact student responses to other queries. Students with prior experience might not confront as many new and challenging realities when participating in ELCIR. Even though the informants were nearly all early career college students $(89.0 \%), 45 \%$ of them $(n=41)$ had traveled internationally. Of those, three $(3.3 \%)$ had previous experience in a study abroad program. The remaining $47(51.6 \%)$ had no prior experience with international travel. With slightly less than half having traveled internationally the potential for new experiences involved with international travel to impact student responses was reduced.

Another topic of interest in the background of the participants was prior experience in a research setting. While this was not included in the evaluation data gathered from 2015 to 2019, it was part of Garcia et al. (2017) investigation. The question was asked of participants in the 2015 pilot of ELCIR. Fourteen of the sixteen respondents had no prior research experience, one reported one academic semester of research and another two semesters.

Another survey question that informs an understanding of the general orientation of participants to ELCIR programming asked about the student's ability to participate without an LSAMPprovided stipend. This question was added for the 2016 survey and retained for the next three years $(n=81)$. Informants were asked which of a series of five responses best described what their "participation in an international research experience would have been without the financial support from LSAMP." The five possible answers were: 1) not participate (55.6\%), 2) probably not participate $(23.5 \%), 3$ ) might have participated (11.1\%), 4) probably participate $(3.7 \%)$, and 5) would have participated (3.7\%). There was also one party who did not respond. Only $7.4 \%$ of respondents felt they would have or probably would have participated without receiving financial support.

\section{Findings Regarding ELCIR Programming}

One of the first queries in the surveys from 2015 through 2019 asked for an overall rating of the ELCIR program. This resulted in a strongly positive response with 49 responses of excellent (53.8\%), 32 of very good (35.2\%), and eight of good $(8.8 \%)$. Two students did not submit a response $(2.2 \%)$ and no responses of fair or poor were received. The median and mode values were excellent.

The remainder of the multiple-choice questions asked of ELCIR participants were discussed in detail in Preuss et al. (2020). Table 4 provides a summary of the findings related to: 1) interest in another similar experience, 2) interest in continued engagement with research, 3) learning achieved regarding the culture of the region visited, 4) increase in knowledge related to the student's major, 5) confidence in travel abroad, 6) impact on interest in employment abroad, 7) impact on career choice, 8) highest degree the student will pursue, and 9) involvement with research post ELCIR. Table 5 chronicles findings related to graduate school: 1) awareness of, interest in, and plans to attend, 2) view of the affordability of graduate school, and 3) perception of support from family regarding a decision to pursue a graduate degree. For each topic considered in Tables 4 and 5, there was no significant difference when comparing responses by gender, ethnicity, race, or prior experience with international travel but measure of the extent of change was not possible as preparticipation data was not gathered.

The responses related to learning and change of perspective can be rank ordered by level of agreement (combining responses of agree and strongly agree).

- Learned about local culture (97.8\%).

- Increased confidence in traveling abroad (96.7\%).

- Interest in another similar experience (95.6\%).

- Encouraged interest in continuing with research (84.6\%).

- Enhanced knowledge in major (78.8\%).

- Increased interest in employment outside the United States (77.8\%).

- Helped with career choice (55.6\%). 
TABLE 4 | Summary of findings from ELCIR surveys 2015-2019.

\begin{tabular}{|c|c|c|}
\hline Topic & $n$ & Finding \\
\hline $\begin{array}{l}\text { Interest in another international research experience like } \\
\text { ELCIR }\end{array}$ & 91 & 87 of the 91 respondents (95.6\%) agreed or strongly agreed; median and mode scores of strongly agree. \\
\hline Interest in continued involvement with research & 91 & 77 students agreed or strongly agreed; median score of agree and mode of agree $(n=40)$. \\
\hline Learned about local culture during time in Mexico & 90 & $\begin{array}{l}88 \text { of } 90 \text { respondents }(97.8 \% \text { ) agreed (strongly agree } n=25 \text {; agree } n=63 \text { ). Only two parties did not agree } \\
\text { (strongly disagree } n=2 \text { ). }\end{array}$ \\
\hline $\begin{array}{l}\text { Knowledge enhanced regarding concepts in field in which } \\
\text { majoring }\end{array}$ & 90 & $\begin{array}{l}78.8 \% \text { agreed (strongly agree } n=32 \text {; agree } n=39 \text { ), } 16.7 \% \text { neither agreeing or disagreeing, and } 4.4 \% \\
\text { disagreeing (disagree } n=3 \text {; strongly disagree } n=1 \text { ). }\end{array}$ \\
\hline Increased confidence in travel outside the United States & 90 & $\begin{array}{l}\text { "Eighty-seven of } 90 \text { students. . Agree or Strongly Agree. ...their ELCIR experience had increased their } \\
\text { confidence in traveling abroad" (Preuss et al., 2020, p. 9). }\end{array}$ \\
\hline $\begin{array}{l}\text { Interest in employment outside the United States following } \\
\text { ELCIR }\end{array}$ & 90 & $\begin{array}{l}77.8 \% \text { agreed (strongly agree } n=47 \text {; agree } n=23), 18.9 \% \text { neither agreeing or disagreeing }(n=17) \text {, and } \\
3.3 \% \text { disagreed (disagree } n=2 \text {; strongly disagree } n=1 \text { ). }\end{array}$ \\
\hline ELCIR helped with career choice & 90 & $\begin{array}{l}\text { "Just over } 50 \% \text { of the students, } 47 \text { of } 90 \text { respondents, agreed" (Preuss et al., 2020, p. 12); } 31.1 \% \text { neither } \\
\text { agree or disagree }(n=28), 13.3 \% \text { disagree }(n=12), 3.3 \% \text { strongly disagree }(n=1) \text {. }\end{array}$ \\
\hline degree will pursue & 81 & $72.8 \%$ indicated would pursue master's or doctorate. \\
\hline Involvement in UR since ELCIR & 81 & 66 said no but 15 said yes just two months following ELCIR. \\
\hline
\end{tabular}

Note: $\mathrm{n}=81$ occurred for questions that were not asked in 2015.

TABLE 5 | Responses regarding graduate school.

Awareness of

Interest in

Plans to attend

Affordability

Family would support decision to go 90
81 "Responses skewed strongly in a positive direction following ELCIR programming with the median value moving up one category, $80.2 \%$ of responses occurring in the top two categories, no responses in the lowest category" (Preuss et al., 2020, p. 10).

81 "Answers skewed positive toward interest in graduate school post-ELCIR. . .all students had heard about graduate school and only six were "Not at all interested...". . a reduction by 28.4 percentage points. The remaining 75 were "A little interested" ( $n=22)$, "Interested" ( $n=29)$, or "Very interested" $(n=24)$. . increases of $125 \%$ for interested and nearly $250 \%$ for very interested" (Preuss et al., 2020, p. 10).

81 Post-participation "a total of $91.4 \%$ of the participants felt that they might, probably would, or would go to graduate school and $43.2 \%$ stated they would go immediately after graduation or at some time in the future. . ..All the other students persisted at their [prior] level of interest or became more interested and none of the students had their level of interest decrease" (Preuss et al., 2020, p. 11).

$9064.4 \%$ agreed or strongly agreed with the statement "I would like to go to graduate school but I just don't see how I can afford it."

$9083.3 \%$ agreed or strongly agreed their family "would be supportive" of their attending graduate school; the "median response. . being Agree" (Preuss et al., 2020, p. 12).

Note: $\mathrm{n}=81$ occurred for questions that were not asked in 2015 .

Fully $72.8 \%$ of these early career minority and first-generation college students indicated intent to pursue a master's degree or doctorate and $18.5 \%$ reported immediate continuation with UR.

ELCIR participation also had a strong impact on awareness of and interest in graduate school when retrospective pre- and post-participation ratings were compared (Table 5) although the use of a nominal scale prevented anything other than descriptive analysis. Like for the responses regarding highest degree the student would pursue, most of the respondents indicated they would attend graduate school with over $40 \%$ stating they would attend immediately after completing their baccalaureate degree. Interestingly, $64.4 \%$ of the respondents expressed concern about the affordability of graduate school while $83.3 \%$ felt their family would support a decision to pursue a graduate degree.

\section{Related Measures in Garcia et al. (2017) Interest in Research and Graduate School}

Garcia et al. (2017) presented ELCIR participants with 14 sentence length descriptions of ways they could engage with graduate school in 2015 and 13 in 2016. Informants were asked to make selections on the pre- and post-participation surveys. In 2015, the "majority... $(n=14)$ indicated they planned to pursue a graduate level degree either in the near future or after obtaining some work experience. The remaining two individuals were uncertain about their future plans" (p. 12). The 2016 respondents $(n=37)$ showed "similar patterns...with a shift towards more research-oriented plans after ELCIR took place. Only two individuals had no plans to go to graduate school, and four were unsure" (p. 12).

\section{Personal and Professional Skills}

Garcia et al. (2017) pre- and post-participation survey included 13 elements grouped as personal and professional skills. The skills listed fell in the following domains: 1) leadership, 2) interpersonal communication, 3) networking, 4) communicating technical information, 5) teamwork, 6) personal management, and 7) construction and presentation of written or verbal research summaries. The rating scale for responses was shifted from a five- to four-point pattern between 2015 and 2016. Due to this, data for the 2 years could not be combined.

Ratings submitted by the 2015 cohort $(n=16)$ showed increases in nine of the 13 areas pre- to post-participation. 
The difference in means was statistically significant at the $p<0.05$ level for one of these, ability "to write a research abstract" (Garcia et al., 2017, p. 9). The authors attributed the general pattern of increase in ratings but few statistically significant differences to high levels of student confidence prior to their international experience.

Pre- and post-participation ratings regarding the same 13 skills were also solicited from the 2016 cohort $(n=37)$. Even though all the pre-participation means were moderately to strongly positive, only four were lower than three (3) on a four-point scale and all the others were between 3.10 and 3.74, there were statistically significant increases for post-participation responses for all 13 at the $p<0.01$ level. This pattern was interpreted as indicating student growth in all seven areas noted above resulting from project programming.

\section{Research Skills}

Garcia et al. (2017) asked about the student's perceived level of knowledge regarding as set of six research activities. The intention was seeking comparative ratings of "level of knowledge. . possessed in the area of research they were working on over the summer" (Garcia et al., 2017, p. 7). The six research activities were knowledge of: 1) "the process of research," 2) "the research literature," 3) "the research skills and/or lab techniques," 4) "how to do statistical analysis of research data," 5) "how to interpret research data," and 6) "how to apply research data" (p. 11). Limiting significance to $p<0.05$, there was one significant finding for 2015. "Students felt that their knowledge of the process of research in their area improved ( $p=0.002)$ " (p. 7). For the 2016 cohort, differences between the pre- and post-assessment means were statistically significant for increases in knowledge at the $p<0.001$ level for all six statements.

\section{Orientation to and Understanding of Culture}

The penultimate topic on Garcia et al. (2017) survey addressed the students' orientation to and understanding of culture. Informants were presented with a list of 19 statements regarding "working and engaging with others of different backgrounds, [and] challenges to their personal beliefs, self-concept, and cultural values" (p. 14). "For the 2015 cohort, there were no significant changes from pre- to posttest. ... The 2016 cohort, however, had many significant differences in their post-test results compared to their pre-test results.... This may be due to...students in this cohort stay [ing] with local families...whereas the 2015 cohort. . .stayed in hotels. This may have had a greater impact on. . cultural perspectives" (Garcia et al., 2017, p. 14).

\section{IMPACT ON ACADEMIC OUTCOMES AND PERSISTENCE}

Garcia et al. (2017) attempted to use Grade Point Average (GPA) as a means of assessing academic impact of ELCIR participation. They compared GPA from the semester preceding the summer experience to that at the end of the semester immediately following it. "There were no significant differences between the two cohorts' overall GPAs from one semester to the next, [but] both [cohorts] demonstrated improvement" (p. 18). Given the level of the GPAs involved, annual averages near or slightly above 3.0, the potential for a substantial improvement in one semester was limited.

The 2017 study also considered persistence. "The retention of both cohorts in the College of Engineering at Texas A\&M University [w] as...remarkable" (p. 18).

- $96.0 \%$ of the 2015 cohort and $88.0 \%$ of the 2016 participants retained majors in Engineering (Garcia et al., 2017).

- $100 \%$ of 2015 and $88.0 \%$ of 2016 participants persisted through their next year as TAMU students (Garcia et al., 2017) when the historic second year persistence rate for TAMU first-time-in-college Engineering students was $86.7 \%$ between 2015 and 2018 (Texas A\&M University, n.d.).

Graduation and persistence data for all LSAMP-supported ELCIR participants between 2015 and 2019 confirm high retention rates and, for the two years for which it is possible, higher than average graduation rates.

- One- and two-year retention rates for the cohort were $97.3 \%$ and $93.0 \%$, respectively, while for their peers in Engineering they were $92.6 \%$ and $87.4 \%$ (Texas A\&M University, n.d.).

- Six-year graduation rates for former participants were $92.3 \%$ when the TAMU rate for undergraduates in the same period was $82.6 \%$ (Texas A\&M University, n.d.).

Student tracking also confirmed higher than average engagement with study abroad programming post-ELCIR, and high levels of graduate school enrollment.

- Forty-one of the ELCIR participants (37.3\%) completed a second study abroad program yet the Open Doors Report on International Educational Exchange indicated only 6\% of engineering students completed study abroad experiences during the same time period (opendoor, n.d.).

- Graduate school enrollment was also high. Thirteen of 33 students (39.4\%) in the 2007-2014 group attended graduate school resulting in nine master's degrees, a $\mathrm{PhD}$, an $\mathrm{MD}$, and two other parties active in graduate study at the time this report was written.

- Seventy-two of the 2015-2019 ELCIR participants completed bachelor's degrees by the time of publication with seven enrolled in or having completed graduate study. Twenty-nine were still TAMU undergraduates and nine were no longer studying at TAMU.

\section{DISCUSSION}

The findings parallel the benefits noted in the literature that were the basis of planning the initiatives. They were consistent for traditional study abroad programming facilitated for junior and seniors and for programming with freshman and sophomores as the audience. 
- Seminars were noted as contributing to student interest in international educational experiences with those prepared and presented by local university personnel showing slightly higher impact.

- Students at all academic levels reported substantial increases in awareness of and interest in graduate school.

- Students at all academic levels and on several different measures reported increases in cultural learning, confidence in travel outside the United States, learning relevant to their major, commitment to continuing involvement with research, interest in another similar experience, and willingness to consider employment outside the United States.

- Approximately $90 \%$ indicated intention to consider graduate school in the future with over $40 \%$ indicating intent to attend immediately following undergraduate study and 39.4\% doing so in the 6 years following participation.

- 37.3\% of ELCIR participants completed a second study abroad experience as undergraduates.

- Participants persisted and graduated at higher rates than their institutional peers.

The outcomes are likely related to the growth in personal, professional, and research skill reported by participants. The areas in which there was the least impact, career plans and immediate continuation with undergraduate research (UR), were measures taken with early career students in the semester following the international experience. It is possible that a significant number did not have firmly established career plans and that there was limited motivation to initiate a new course of UR while completing the final requirements of the ELCIR program. Having $18.5 \%$ of the students continue with UR immediately is positive as "approximately $25 \%$ of TAMU engineering students participate in UR prior to graduation" (Preuss et al., 2020).

The TAMUS LSAMP findings included no statistically significant differences regarding student experience, learning, or perspectives when compared by gender, ethnicity, race, and prior experience with international travel. This is a notable confirmation that the programming described was efficacious for college students from underrepresented groups, many of whom were first-generation college students who may also have had limited resources. The vast majority of the students indicated they would not have been able to participate without financial assistance and nearly two-thirds indicated uncertainty regarding whether and how they could afford graduate school. Yet, it is also notable that over $84 \%$ of informants, who were predominantly URM students with $91.1 \%$ identifying as Hispanic, felt their family would support pursuit of a graduate degree as this does not align with historic patterns in STEM graduate programs and employment (Bayer Corporation, 2012; Linley and George-Jackson, 2013; Collins, 2018; NSF, 2018).

That these findings existed for URM students early in their academic careers and as juniors and seniors, across thirteen years of activity, with students attending Minority-Serving Institutions and a Predominantly White Institution (PWI) with differing Carnegie classifications, and for two different Diversity/Global Learning frameworks distinguishes this material from that describing other efforts. As a combined set, the data demonstrate substantial impact on participant perspective, interest, intention, and learning as well as suggesting a connection to high levels of persistence, continuing engagement with Diversity/Global Learning programs, degree completion, and enrollment in graduate school.

\section{IMPLICATIONS}

There are several simple implications of the findings. First, both traditional study abroad programming and structured programming including short-term involvement in research in an international setting were efficacious with LSAMP participants. Programming of either type is likely to have similar results at other institutions as the TAMUS participants came from a PWI, an HBCU, and an HSI that have different Carnegie classifications. Second, both types of programming were effective with students from underrepresented groups and who were first generation college students. Third, the year in school of the participant does not appear to influence the potential for substantial benefit from the programming, although this is a generalization based on different age groups reporting similar results to the two different approaches rather than samples of students at every level of undergraduate study experiencing both approaches. An important caveat, though, is the ability of an institution to provide stipends to enable student participation as this was a primary component in both approaches enacted by TAMUS LSAMP.

Replication of the initial pedagogical framework is possible for many institutions. Identifying funds for participant stipends would be the most challenging element. Sponsoring personnel could use existing study abroad programming as the platform and collaborate with their study abroad office in refining informational and orientation seminars for participating students. Replication of ELCIR requires funding to support participants, creation of a one-unit course, identification of and program planning with an institution of higher education outside the United States, and planning and implementing pre- and post-participation seminars and processes. Garcia et al. (2017) and Preuss et al. (2020) provide additional details as can authors of this publication. While substantial institutional and time commitment would be required to initiate and maintain such an effort, it may be more possible for institutions near the international borders of the United States and the potential for long-term societal impact demonstrated by the pronounced and positive outcomes for URMs has a strong appeal in light of the known need for persons with STEM degrees and patterns of underrepresentation in the STEM workforce of the United States employment (Bayer Corporation, 2012; Linley and George-Jackson, 2013; Collins, 2018; NSF, 2018). 


\section{LIMITATIONS}

The data came as student self-reports and addressed experience, self-assessment, and personal opinions. Control groups were not included. Thus, the majority of information considered herein is descriptive. Inferential analysis was limited to one data stream, although those findings corroborated and elucidated the descriptive analysis. The sample sizes were moderate, less than 100 persons. The findings are then, primarily, a descriptive case study of two patterns of Diversity/Global Learning programming. Support from other sources and through further investigation by TAMUS LSAMP will be necessary to establish more robust evidence of efficacy.

\section{DATA AVAILABILITY STATEMENT}

The datasets presented in this article are not readily available because the data include responses from a small number of participants. This could make information individually identifiable. Requests to access the datasets should be directed to exquiri.michael@gmail.com.

\section{ETHICS STATEMENT}

The studies involved human participants and were reviewed and approved by the Texas A\&M University Institutional Review Board. The participants provided their written informed consent to participate in this study. Written informed consent was obtained from the individuals for the publication of any potentially identifiable images or data included in this article.

\section{AUTHOR CONTRIBUTIONS}

First authorship is shared by MP, SM, and JA. MP compiled applicable data from evaluation materials, completed quantitative data analysis, planned and led group analysis of qualitative data, completed secondary research, and drafted the article. SM and JA

\section{REFERENCES}

American Association of Colleges and Universities [AACU] (n.d.). High Impact Educational Practices. Available at: https://www.aacu.org/node/4084 (Accessed February 1, 2021).

Borri, C., Guberti, E., and Melsa, J. (2007). International Dimension in Engineering Education. Eur. J. Eng. Educ. 32 (6), 627-637. doi:10.1080/ 03043790701520586

Brux, J. M., and Fry, B. (2010). Multicultural Students in Study Abroad: Their Interests, Their Issues, and Their Constraints. J. Stud. Int. Educ. 14 (5), 508-527. doi: $10.1177 / 1028315309342486$

Chan, A., and Fishbein, J. (2009). A Global Engineer for the Global Community. The J. Pol. Engagement 1 (2), 4-9.

Collins, K. H. (2018). Confronting Color-Blind STEM talent Development: Toward a Contextual Model for Black Student STEM Identity. J. Adv. Academics 29 (2), 143-168. doi:10.1177/1932202X18757958 coordinated data gathering from partnering institutions and completed analysis of those data, completed data gathering and analysis specific to TAMU, helped plan the presentation, and provided comments on drafts. Senior authors are KB-P, KW, SW, PO, FP, JM, and MR. They coordinated site-specific activity and commented at different points during development of the article. JK, HL, $\mathrm{MA}$, and SG are last authors. JK and HL planned and conducted evaluation processes that resulted in data presented in the article. MA and SG coordinated the ELCIR efforts, approved use of content from their 2017 publication, gathered institutional data, and commented on descriptions of ELCIR in this article.

\section{FUNDING}

This material is based upon work supported by the National Science Foundation under Grants No. HRD-073290, 1304975, and 1911375. Any opinions, findings, and conclusions or recommendations expressed in this material are those of the author(s) and do not necessarily reflect the views of the National Science Foundation.

\section{ACKNOWLEDGMENTS}

We would like to acknowledge the assistance of Yahaira Franco and Jessica Hernandez from Texas A\&M International University with coding evaluation themes related to the paper. We would like to acknowledge Mahmoud Khasawneh, John Kilburn, and Jared Dmello for their leadership and involvement with the LSAMP efforts on the TAMIU campus. We would also like to acknowledge Zenon Medina-Cetina for his efforts initiating the ELCIR program and continued mentoring of and engagement with ELCIR participants. Content included in the presentation above from Preuss et al. (2020) was used with the permission of the authors and the publisher.

Corporation, Bayer. (2012). Bayer Facts of Science Education XV: a View from the Gatekeepers - STEM Department Chairs at Americas Top 200 Research Universities on Female and Underrepresented Minority Undergraduate STEM Students. J. Sci. Educ. Techn. 21, 317-324.

Dwyer, M. M., and Peters, C. K. (2005). The Benefits of Study Abroad: New Study Confirms Significant Gains. Chicago, IL: Institute for the International Education of Students. Available at: http://www2.clarku.edu/offices/studyabroad/pdfs/IES\% 20Study.pdf (Accessed February 2, 2021). doi:10.1002/0471667196.ess1248

Garcia, S. J., Alves, M. C., Pariyothorn, M., Myint, A., and Hardman, A. K. (2017). ELCIR - Engineering Learning Community Introduction to Research: a Research and Global Experience Program Supporting First Generation Low Incoming Underrepresented Minority Students. American Society for Engineering Education Papers \#20476. 1-24.

Graham, J. M., Caso, R., and Rierson, J. (2001). The Effect of the Texas A\&M University System AMP on success of Minority Undergraduates in Engineering: A Multiple-Outcome Analysis. Proc. American Society for Engineering Education Conference \& Exposition, 1-12. 
Indiana University Center for Postsecondary Research (n.d.). The Carnegie Classification of Institutions of Higher Education. Available at: https:// carnegieclassifications.iu.edu/index.php (Accessed February 1, 2021).

Kamdar, N., and Lewis, T. (2015). Deriving Long-Term Benefits from Short-Term Study-Abroad Programs. J. Manag. Eng. Integration 7 (2), 1-10.

Kolb, S. M. (2012). Grounded Theory and the Constant Comparative Method: Valid Research Strategies for Educators. J. Emerging Trends Educ. Res. Pol. Stud. 3 (1), 83-86.

Kuh, G. D., and O'Donnell, K. (2013). Ensuring Quality and Taking High Impact Practices to Scale. Washington, DC: American Association of Colleges and Universities.

Linley, J. L., and George-Jackson, C. E. (2013). Addressing Underrepresentation in STEM fields through Undergraduate Interventions. New Dir. Student Serv. 2013, 97-102. doi:10.1002/ss.20073

Merriweather, S. P., Lamm, H. A., Walton, S. D., Butler-Purry, K. L., Kelley, J., ThomassonE, K., et al. (2017). TAMUS LSAMP Project: 25 Years of success Finding and Implementing Best Practices for URM STEM Students. American Society for Engineering Education Papers \#18491, 1-20.

National Science Foundation (2018). 2016 Doctorate Recipients from US Universities. Available at: https:/www.nsf.gov/statistics/2018/nsf18304/static/ report/nsf18304-report.pdf (Accessed February 1, 2021).

Opendoor (n.d.). Top fields of Study of U.S. Study Abroad Students. Availableat: https://opendoorsdata.org/infographic/top-five-major-fields-of-study-of-us-study-abroad-students/ (Accessed May 21, 2021).

Paras, A., Carignan, M., Brenner, A., Hardy, J., Malmgren, J., and Rathburn, M. (2019). Understanding How Program Factors Influence Intercultural Learning in Study Abroad: The Benefits of Mixed-Method Analysis. Frontiers 31 (1), 22-45. doi:10.36366/frontiers.v31i1.441

Potts, D. (2015). Understanding the Early Career Benefits of Learning Abroad Programs. J. Stud. Int. Educ. 19 (5), 441-459. doi:10.1177/1028315315579241

Preuss, M. D., Merriweather, S. P., Walton, S. D., and Butler-Purry, K. L. (2020). "International Research Exposure: Impact on Early-Career, Undergraduate Engineering Students," in Proceedings of IConSES 2020- International
Conference on Social and Education Sciences. Editors V. Akerson and I. Sahin (Chicago, IL, USA: ISTES Organization), 1-15.

National Center for Education Statistics (2018). Enrollment and Degrees Conferred in Hispanic-Serving Institutions, by Institution Level and Control, Percentage Hispanic, Degree Level, and Other Selected Characteristics: Fall 2018 and 2017-2018. Available at: https://nces.ed.gov/ programs/digest/d19/tables/dt19_312.40.asp?current=yes

Smith, D., and Mitry, D. (2008). Benefits of Study Abroad: The Case for ShortTerm Programs. J. Res. Innovative Teach. 1 (1), 236-246.

Texas A\&M University (n.d.). Data and Research Services: Retention and Graduation. Available at: https://dars.tamu.edu/Data-and-Reports/Student/ Retention-Graduation (Accessed February 09, 2021).

U.S. Department of Education (2020). White House Initiative on Historically Black Colleges and Universities. Available at: https://sites.ed.gov/whhbcu/onehundred-and-five-historically-black-colleges-and-universities/ (Accessed February 1, 2021).

Conflict of Interest: Author MP was employed by the company Exquiri Consulting, LLC.

The remaining authors declare that the research was conducted in the absence of any commercial or financial relationships that could be construed as a potential conflict of interest.

Copyright (c) 2021 Preuss, Merriweather, Avila, Butler-Purry, Watson, Walton, Obiomon, Pezold, Murry, Roth, Kelley, Lamm, Alves and Garcia. This is an openaccess article distributed under the terms of the Creative Commons Attribution License (CC BY). The use, distribution or reproduction in other forums is permitted, provided the original author(s) and the copyright owner(s) are credited and that the original publication in this journal is cited, in accordance with accepted academic practice. No use, distribution or reproduction is permitted which does not comply with these terms. 